\title{
RE VIS T A
}

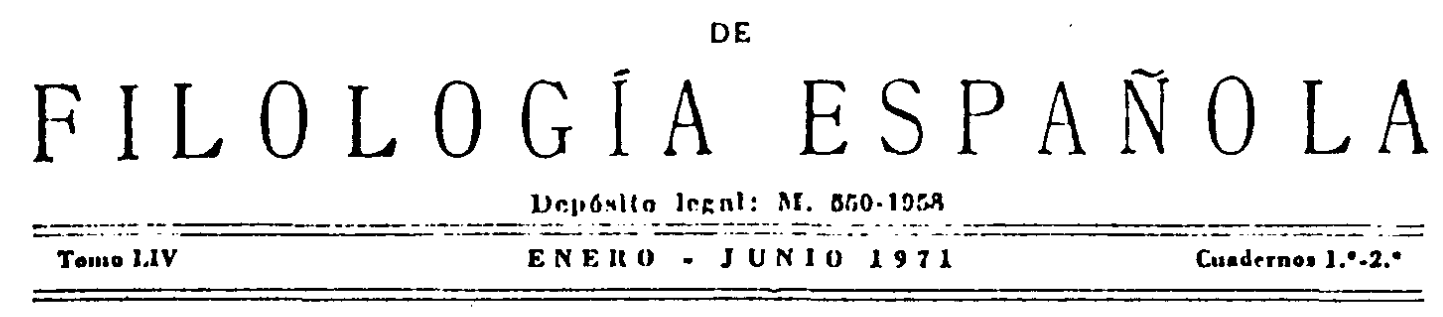

CANTARA Y CANTASE

I. El problema.

I.I. En todas nuestras gramáticas, al igual que en su modelo normativo 1a Gramática de la Academia, encontramos en el paradigna de la conjugación o flexión verbal, y en el llamado imperfecto de subjuntivo, dos formas en disyunción: cantara o cantase ${ }^{1}$. Y nos preguntamos: ¿se trata de una simple alternancia formal, de libre elección onomasiológica, de uso aleatorio? $¿()$ estamos ante un empleo regulado y sistematizado, con diferente valor funcional en la estructura verbal?

Vamos a intentar clar una respuesta coherente a ese interrogante, a menudo caballo de batalla en la descripción verbal del español y problema que está sin resolver en las gramáticas descriptivas de nuestra lengua, dirigidas a nativos o a extranjeros.

I.2. Examinemos antes los trabajos ${ }^{2}$ que diversos gramáticos, en mayor número extranjeros, han dedicado al empleo de las formas -ra y -se de nuestro subjuntivo.

La revista Hispania de California se preocupó del tema ya en I925 y 1926 primero y más tarde en 1947 y $19+8$ principalmente, con una serie de artículos de diferentes profesores. G. I. Dale ${ }^{3}$ deduce la preponderancia del -ra sobre el -se a través de un corpus de formas tomado de la revista Interaniérica -ique era redactada en inglés y traducida luego por dos sudamericanos! - Es la critica que le hace M. IV. Graham ${ }^{4}$, quien, a su vez, basa su razonamiento únicannente en textos literarios

1 Rien, Acaulimia lisprĩola, Gramática de la lengua española. Madrid, Ispasa-Calpe, I 962, 95, a) b) c). Y esto según su última mueva edición, reformada, de r93 In, pues hasta I9r 7 incluida también en ese imperfecto de subjuntivo la forma cantarta.

2 Impleamos la bibliografia reunida a través de varios años de preocupación por el verbo, más amplia que la importante ofrecida por R. NAvas Rulz: Bibliografta crilica sobre el subjunlivo español. Madrid, CSIC., I 968. Actas del XI Congreso Internacional de I,ingüistica y I’ilologia Rominicas, v. III, pp. 1.823-1.839.

- G. I. Dal1:: The Imperfed Subjunctive. H, VIII, 1925. P1. I 27-I 29.

1 M. W. Graina: The Imperfect Subjunctive in Spain America. $H, \mathrm{IX}$, I926, PP. 4 6-49. 
para llegar a la misma simple conclusión de mayor empleo de -ra que de -se.

Lis $I_{\text {. }}$ O. Wright el que mejor investign el problema cn varios artículos. Lin uno de ellos ${ }^{1}$ distingue el habla culta de la menos culta y observa cierto valor funcional en esta alternancia de formas, aunque no llega a sistematizarla. Vuelve sobre el tema en $I_{947^{2}}{ }^{2}$ donde tampoco sistematiza, pues se limita a enumerar una serie de 30 posibles empleos de -ra, que considera todos ellos correctos, incluyendo en su lista la forma $-r a$ de indicativo. J. Mallo le ataca en lo que se refiere a este último valor ${ }^{3}$ con un razonamiento normativo. Tilda a esta forma -ra de indicativo de sproducto de la afectación y del desconocimiento de la gramátican , pero tampoco nos propone soluciones ni sistematización general ${ }^{5}$. D. L. Bolinger publica un artículo apaciguador distinguiendo el doble diferente enfoque en ellos ${ }^{\circ} \mathrm{y}$ ocho años después pone en duda la libre altermancia, afirmando que -ra es más desiderativo que $-s e^{\text {? }}$.

1.3. Recorremos brevemente esta bibliografía, pues muy poca información obtenemos de estos artículos, ya que en ninguno de ellos se lia estudiaclo el problema con un criterio totalizador ni se ha buscado la respuesta en una explicación inmanente con un método colerente dentro de la estructura funcional del sistema.

E. Alarcos Llorach, en un enfoque más amplio y sistematizado, asegura: "En la lengua moderna, las dos formas existentes: canlara y canlase son perfectamente equivalentes, no sólo en el sistema, sino en el uso linginisticon" 8 .

Pero nos preguntamos: ¿son formas verdaderamente equivalentes? Si lo son efectivamente, ¿cómo es posible que se conserven con tanta vitalidad dos formas del sistema para exactamente la misma función? ¿Por qué no reacciona la economía de la lengua?

1 I. O. IVRIGnit: Futher Notes on tra and -se. H, IX, I926, p. 20 .

2 IDIiar: The spanish verb-form with the grealest variety of finclions. $I I, \mathrm{XXX}$. 1947. 1)1. 4 \$8-495.

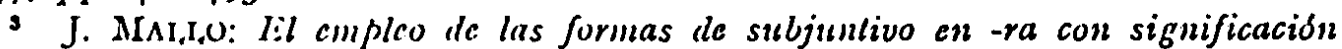
de tiempos del indicalivo. $I I, \mathrm{XXX}, 1947,1 \mathrm{p} .484-487$.

4 IDEM: p. 485 .

- Obsérvese que, según veremos más adelante, nosotros distinguimos y precisamos el valor de cantara = habia cantado, pero no admitimos su valor de equivalente sustituto de cante, coincidiendo asi con la Academia: Gramática..., $c p$. cit., 3or, nota.

- D. I. J3oringir: On the -ra form. $H, \mathrm{XXXI}$, I948, pp. 34I-342.

7 Inlis: Subjuntive -ra and -se: Free Varialion? $H, \mathrm{XXXIX,} \mathrm{1956,} \mathrm{pp.}$ $3+5-3+9$.

- 1:. Alakcos Ia.oracit: Sobre la estructura del verbo español. B BMP. I949. pp. 50-S 3 . I aliora en Esludios de gramdica funcional. Madrid, Gredos, 1970, p. 68. 
2. Un corpus.

2.I. En tun intento de esclarecer el problema licmos remido un corpus de trabajo, un conjunto de 250 formas en -ra y en -se, espigadas a través de textos que han pasado ante nucstros ojos o han llegado directamente a nuestros oídos. Lin esta recopilación de empleos concretos de español, hemos procurado apartarnos de los textos puramente literarios por su menor espontaneidad, a fin de poder cncontrar más detalles de la tendencia seleccionadora del sistema'. Iil resultado es el siguiente ${ }^{2}$ :

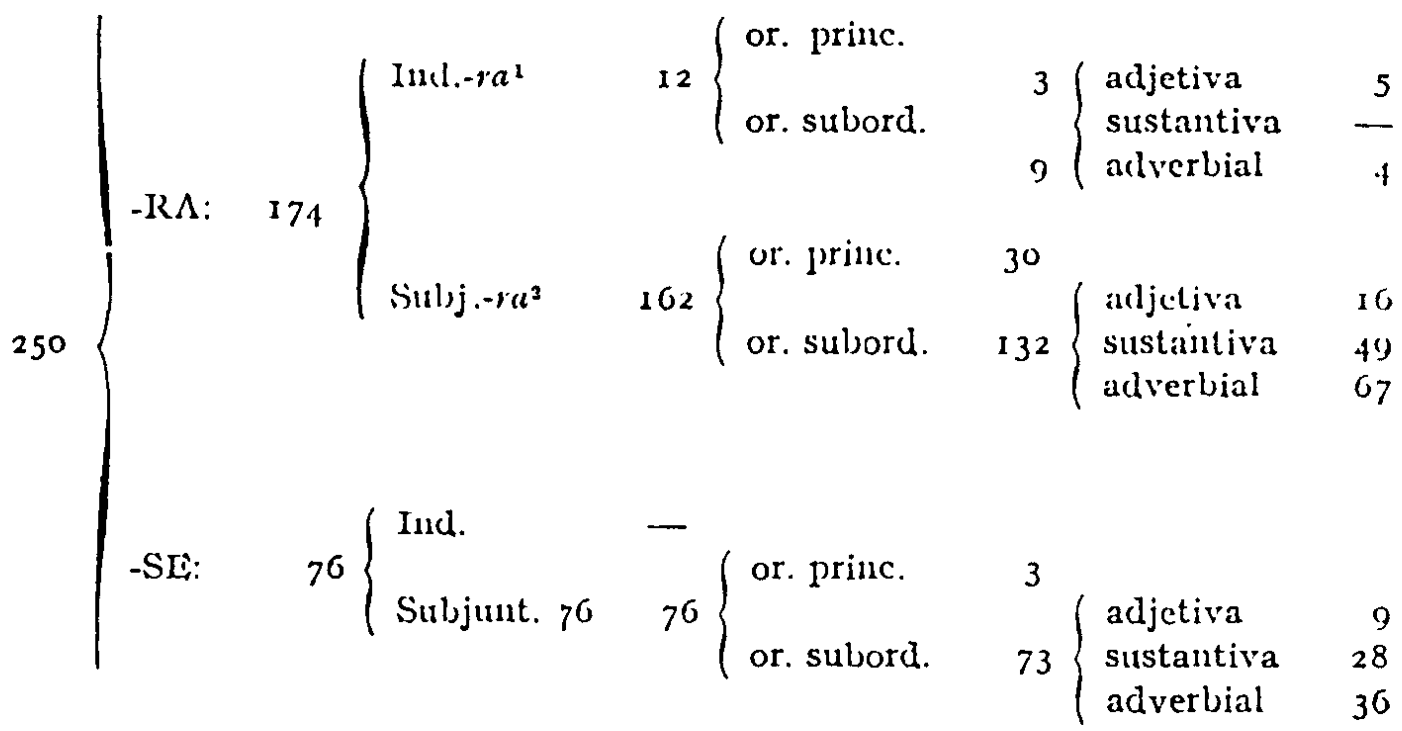

2.2. Vamos a comentar esta estadística. Es patente, en primer lugar, el mayor empleo de formas en $r a$ con una preponderancia neta, frente a las formas en $-s e^{3}$. Incluso si eliminamos de la comparación los doce empleos de $-r a^{1}$ de indicativo, observamos en el imperfecto

1 Ifemos tomado estas 250 formas en -ra o -se de cmpleos concretos del habla ch el lenguraje oral, usos espontíneos, o en la sección de sucesos y noticias auodinas de los periúxlicos, textos en principio muy poco corregidos, en los que el hablante nativo va más influido por las fuerzas inmanentes de la forma interior de la lengua y, por ello, mucho nás oportunos, en muestro criterio, que los textos literarios, para observar la tendencia seleccionadora y reorganizadora de la lengua en su estructura funcional. Aunque también daremos ejemplos literarios que, fuera de la cucuesta, corroboran los resultados del habla, ya sca popular ya culta.

2 Diferenciamos $-r a^{1}$ con valor de indlicativo, de $-r a^{2}$ con valor de subjuntivo, según lo hemos heclio en otro trabajo, cfr. V. Lamiguiz: El sistema verbal del español actual. Intento de estruchuracion. Revista de la Universidad de Madrid, 1969, XVIII, 69, I, pp. 24 I-265.

3 Nótese la coincideucia con los artículos citados en ap. I.2. 
de subjuntivo $I 62-r a^{2}$ frente a $76-s e, 68 \%$ y $32 \%$ respectivamente, que contradice la creencia de S. Gili Gaya de que "en España predomina actualnente $-s e$ en la conversación ordinaria" ', aunque añade ura se usia mucho entre personits cultas y en lat lengua cscritan"

No hay muchos años de separación entre nuestro gramático S. Gili Gaya y nosotros. Pero consideremos lo que ha ocurrido en Hispanoamérica. A. Bello, cuya Gramática apareció en 1847 , cree que la forma en -se "es de mucho más frecuente uson" ${ }^{3}$. Y. su apreciación está de acuerdo con el empleo mucho más repetido de la forma -se que -ra en la redacción de su propia Gramática. Mientras que Ch. E. Kany afirma en 1963: "la forma en -se casi ha desaparecido del habla de la mayor parte de Hispanoamérican" ${ }^{4}$. Ante estos datos, nos atreveríamos a insinuar un aumento en el habla actual de la forma en -ra correspondiente a una disminución de la forma -se en Tispaña, al igual que lo ocurrido en Hispanoancricica.

2.3. Si observamus el modo de la cnchesta, vemos que la forma -ra puede ser de subjuntivo o de indicativo, mientras que la forma -se siempre es del subjuntivo.

Nadie niega el valor $-r a^{2}$ de subjuntivo. $\mathrm{Y}$ el valor $-r a^{1}$ de indicativo está suficientemente probado. En su diacronfa, «la forma $-r a$ procede del pluscuamperfecto de indicativo latino, cuya significación conservó en castellano hasta el siglo xvir, en que cayó en desuso, pero no del todo, pues se lialla también alguna vez con dicho valor en escritores del siglo Xviri” ${ }^{5}$. Además, podemos fundamentarnos en la valiosa obra, perfectamente documentada, de L. O. Wright ${ }^{6}$, que coincide con la afirmación de la Academia y añade la abundancia de su empleo en el siglo xix. I en sincronía actual puede justificarse "por el criterio lingüístico de 'casilla vacía' en el sistema verbal, que sin esta forma estaría en desequilibrio con cinco formas simples en indicativo. De ahí la tendencia posible lacia el equilibrio, que scĩalamos al introducirla" ?. I es fácil documentarla: frecuente en hablantes hispanoame-

1 S. Gili GaYa: Curso superior de sintaxis española. Barcelona, Vox, 1964, ap 137. Insistimos en el hecho de que nuestra encuesta es eminentemente conversacional, cfr. nota I de la p. 3 .

2 I BIDEM.

- A. Betro: Gramática de la lengua castellana. Caracas, 1951, §655.

- Cir. L. Kany: Sintaris hispanoamericana. Madrid, Gredos, r969, p. 222. Ia celición original en ingles apareció en I963.

- Rrar, Acadenia Iispañor,a: Gramática..., op. cit., § 300, a).

- I. O. W'riguT: The -ra verb-form in Spain. Berkeley, 1932.

$\checkmark$ V. I.AMrQuiz: El sistema verbal..., op. cit., § 2. 4. 2. 
ricanos; en hablantes peninsulares, especialnente gallegos, es verdad; diarianente en lenguaje periodístico; y en literatura actual:

I. ${ }^{0}$ en escritores gallegos: "Para tun scrviclor, que recogicra sus últimas palabras de arrepentiniento con el nismo gozo con que recogicra la más doradia mies el labrador, no doja de ser fuerte impresion la lectura de lo escriton (C. J. Celia).

2.0 en escritores castellanos: "Vestía el traje que le confeccionara 'Téllez el sastre en I94I, y la corbata de picué agrisada que le regalura Lucita" (MI. Delibes).

Iin cambio, la forma en -se siempre es subjuntivo. A este propósito debemos mencionar las formas -se indicativas de que nos habla V. Bejarano ${ }^{1}$, paralelas al empleo hispanoamericano que cita Cli. Li. Kany ${ }^{2}$. Pensamos que se trata de comunes uitracorrecciones ${ }^{3}$ que $n o$ pueden tenerse en cuenta en el sistena. Linguiisticamente es preciso distinguir, por un lado, los $I 8$ casos de -se indicativo que V. Bejarano ha documentado en La sombra del ciprís es alargada (le M. I) clibes. J,o consideramos como un rasgo (eintencionado?, no lo sabemos) de tul alutor en una de sus obras ${ }^{4} ; y$, por otra parte, los otros dos casos que cita, así como los que aduce Ch. Li. Kany, pueden catalogarse como esporádicos. Incluso pensamos que introducirlos en un razonamiento de sistematización, tal la que intentanos, sería anticientífico.

2.4. Veamos ahora el empleo de las formas que estudiamos, en oración principal o independiente:

$-r a^{1}$ de indicativo: documentada 3 veces, pero clebemos precisar que en los tres casos ha sido en boca de hablante gallego: «N Fucra Pedrito el que organizara la broma; fuera genial" (Oral.), coincidiendo con Ch. E. Kany, quien en oración principal lo atribuye a ucscritores gallegos o asturianos» ${ }^{5}$.

$-r a^{2}$ de subjuntivo: 30 empleos en oración principal, frente a $-s e$ de subjuntivo: 3 empleos en oración principal.

Lis preciso distinguir ell $-r a^{2}$ :

1 V. Biojarano: Sobre las dos formas del imperfecto de subjunliio y el empleo de la forma -se con valor de indication. Salaninanca, STRLiNAL, IgGz.

2 Cit. I.. INANY: Sintaxis..., op. cil., p. 213.

3 Cfr $\$ 3.4 . y \S 4.2$.

- Lis, en efecto, excepcional pues no se da ese rasgo en sus dentás obras. Como numestra concreta, he aqul el resultado de un escrutinio exlanistivo de La morlaja, Madrid, Alianza Liditorial, I97o: wu -ra ${ }^{1}$ en p. 45: 26 formas de $-r a^{2}$ cll pp. 37, 37, 43, 44, 44, 45, 46, 48, 50, 59, 59, 61, 62, 62, 62, 64, 67, 68, 69, 73, 76, 78, 79, y 79; con io formas cil -se en 1p). 40, 42, 43, 44, 44, 46, 50, 52, 57, 59, $61,62,62,62,68,75$ y 78 .

- Cir. E. Kany: Sintaxis..., op. cil., p. 209. 
I. ${ }^{\circ}$ Si se trata del último grado de opinión subjetiva o de evasión de cortesía en el funcionamiento de los niveles de actualidad ${ }^{1}, 2$ I casos de los 30, va sin introductor y no alterna con -se ": "Quisiera amistad, con fines matrimoniales, con señorita o viuda sin hijos» (Pueblo, 9-2-68.)

2.0 Si se trata de un empleo con valor funcional de significación subjuntiva, los 9 casos restantes de los 30 , lleva introductor y alterna con -se: "Se encuentra cu la Costa $\Lambda z u l$ iquién pudiera!, ¿verdad?, amigo" (Pueblo, 26-I-68.); “¡jalá pudiese ir yo también!» (Oral.)

Creemos que es importante insistir en ese introductor que permite la alternancia de formas, en relación con el diferente valor que luego sistematizaremos ${ }^{3}$, y es marca léxica que diferencia esos dos distintos valores en el imperfecto de subjuntivo.

2.5. Consideremos el comportamiento de las formas en oración subordinada o dependiente. En cuanto al -ra ${ }^{1}$ de indicativo, no la hemos docunentado en subordinada sustantiva, al igual que Ch. I. Kany 4 I a razón pucle ser para cvitar una anfibología en la marca de época, scgún explicaremos ${ }^{5}$. Sí encontramos esta forma, 5 casos cll nuestra encuesta, en subordinada adjetiva: "Aquí el Cáliz de ágata que Cristo usara en la íltima cena" ( $A B C, I 6-3-60$.$) ; y 4$ casos en subordinada adverbial: "I,o prueba el epitafio de Antonio Machado, cuando escribiera aquello de "Nadie esta lira taña..." (Pueblo, 9-2-68.)

Todos estos empleos de $-r a^{1}$ se caracterizan por una visión lacia el pasado. liste enfoque hace difícil precisar si equivale a thabía cantado" o a "canté", con el consiguiente problema normativo ", que se resuelve únicamente por la correspondencia de tiempos.

2.6. Ein cuanto a las formas de subjuntivo $-r a^{2}$ y $-s e$, se encuentran en los tres tipos de subordinadas, en alternancia y preponderando $-r a{ }^{2}$, I 32 casos, scbre -se, 73 casos. Damos, como muestra, los correspondientes pares de ejemplos:

I. E En subordinada adjetiva: "El que llegara el primero, tenía que telefonearle" (Oral.) "lil era así y no había quien lo cambiase" (Oral).

2.0 Iin subordinada sustantiva: "¿Cómo se explica que el vagabundo escapara tan rápidamente?" (Pueblo, I4-2-68.) "Le ordenó que se fuese inmediatamente» (Oral.)

1 Cfr. V. IAamquiz: El sistema verbal..., op. cit., § 2. 4. 4. Idem: Los niveles de actualidan, Revista Española de Lingiiistica Madrid, I, I, pp. 89-96.

2 Cfr. $\$ 3.3$.

3 Cfr. $\$ 3.4$.

- Cul. Li. Kand: Sintaxis..., op. cit., p. 209.

- Cfr. \$ 3. 2.

- Cfr. $\$$ I. 2., nota 9. 
3. In subordinada adverbial, los dos ejemplos con igual valor final: "Los donó en vida el autor para que pudieran ser utilizados por los investigadores» ( $A B C, 6-2-68$.$) "Y para que nada faltase, lograron$ dos goles prodigiosos" (Pucblo, 30-I-68.)

3. Finucionamicnlo de la cslruclura.

3.I. Si relacionamos los resultados comentados del corpus de la encuesta con el funcionamiento de la estructura, podemos considerar la marca que priva, al distinguir tres casos en el comportamiento de las formas $-r a$ y $-s e$, a fin de dar una explicación lingïística al funcionamiento de estas dos formas del imperfecto de subjuntivo de nuestras gramáticas:

I. Oposición $-r a^{1} /-r a^{2}$.

2.0 Iiorma $-r a^{2}$ sin alternancia con $-s c$.

3. Oposición -se/-ra ${ }^{2}$.

3.2. Lin la oposición $-r a^{1} /-r a^{2}$, funciona la marca de modo, que expresa el grado de realización de la acción verbal, vista por el hablante en enfoque subjetivo: el indicativo, $-r a^{1}$, marcado, se opone al subjuntivo, $-r a^{2}$, no marcado.

Coincide con esta marca de modo, la marca de época. La forma -ra ${ }^{1}$ ofrece una prospección hacia el pasado, marcado, mientras que - $r a^{2}$ lleva una visión hacia un presente inactual, no marcado por ser presente y por ser inactual ${ }^{1}$. Véase la oposición con marcas de época y modo en este doble ejemplo literario que la documenta:

-ra ${ }^{1}$ : "Iill transatlántico que trajera de Méjico al Indiano había anclado a primera hora de la mañana frente al rompeolas» (J. A. Zunzunegui.)

$-r a^{2}$ : "Migucl se sentía un poco inquieto ante la idea de que Ivonne desperlara y cncontrara a aquella muchacha sentada en la alfombra" (J. M. Gironella.)

3.3. Lil segundo caso trata de la forma $-r a^{2} \sin$ alternancia con -se, como en el cjemplo: "Aqquélla del notario quisiera yo ahora" (A. M. Lera.)

Ya A. Bello había anotado este empleo exclusivo de $-r a^{2}$, detalle importante olvidado por la gran mayoria de las gramáticas tradicionales. Su valor es de presente inactual de subjuntivo y se emplea, según hemos

1 Cfr. $\S 2.5 . y \S 3.3$.

2 A. BliLl,o: Gramática..., op. cit., $\S 695$. 
visto ${ }^{1}$, para expresar el último grado de evasión de cortesía o de opinión subjetiva. Va condicionado al uso en oración independiente sin introductor; y en cuanto al lexema, queda restringido esencialmente a querer, poder y deber.

I, a época de presente inactual de esta forma en $-r a$, como todo $-r a^{2}$, ofrece una prospección que se sitúa en la "construcción de futuro" de que dispone todo presente, siguiendo a $G$. Guillaume ${ }^{2}$, o, si se preficre, va en un co-futuro de la terminología de $A$. Bello, que viene a ser lo mismo ${ }^{3}$.

3.4. I el tercer caso trata de la oposición -se/-ra .2 En ambas formas el modo es idéntico: subjuntivo. La época puede servir ya de marca pertinente: -se, pasado, marcado, en oposición a -ra, presente, no marcado 4 .

Pero pensamos que el nivel de actualidad desempeña un papel importante en esta oposición, a la cual sirve de marca funcional: -se, nivel actual, narcado, frente a $-r a$, nivel inactual, no marcado. Esta marca caracteriza con visión subjuntiva al -se actual, pues "la forma en -se es verdaderamente subjuntivan ${ }^{5}$, frente al $-r a$ inactual con visión optativa o valor clesiderativo ${ }^{\circ}$.

Recordemos para cxplicar esta marca de actualidad que en el subjuntivo latino, y a través del latín en el subjuntivo de las lenguas románicas, se fundieron los modos subjuntivos y optativo del indoeuropeo. No es extraño que un prestigioso indoeuropeísta, E. Benvéniste, nos haya hecho ver en el verbo los niveles de actualidad?, marca de esta oposición -se / -ra, que hace salir a flote esos dos antiguos modos.

1 Cfr. ap. 2. 4. I a marca pertinente de los diferentes pasos en la evasión, véase en V. I.amiQuiz: El sistema verbal..., op. cit., \$2. 4.5.

2 G. Guil,

3 Por todo esto, no podemos estar de acuerdo con B. Potrier: Gramálica del español. Trad. esp. A. Quilis. Madrid, Alcalá, 1970, p. I18, donde nos parece según la disposición del gráfico en su $N$. B., que considera este -ra como un -ra pues este últino lleva un evidente enfoque de sentido contrario, hacia el pasado, como licmos visto en $\$ 3.2$.

- I’ara la época respectiva, cfr. V. LAmiquiz: El sistenla verbal..., op. cit., § 3. 3.

- Risal ACAviamin Lispañora: Gramática..., op. cit., $\S 300$, e). 'Tan vercladeranente subjuntiva, en efecto, que esta forma en -se numca desempeña función cle indicativo: cfr el resultado de nuestra encuesta en el $\S 2$. r. y la explicación de los casos de ultracorrección en el $\S 2$. 3 .

- Cfr. $\$$ I. 2.

7 Cfr. I: Bninvíniste: Les relations de temps dans le verbe franf̧ais en Problimes de linguistique générale. Parts, Gallimard, I966, pp. 237-250. Y sus consecuencias de sistematización verbal, cfr. V. LamiQuiz: El sistema verbal..., op. cil., § 2. 4. I' su aplicación lingüistica y literaria, cfr. H. WuINRIcn: Estructura y funcion de los tiempos en el lenguaje. Mradrid, Gredos, 1968, passim. 
Teniendo en cuenta el sentir de la Academia, cuando dice que esas dos formas "no son enteramente equivalentes" ${ }^{1}$, coincidimos con el criterio de los gramáticos que atribuyen a la forma - $r a$ un valor desiderativo o mayor inclinación a la hipotética realización de la acción verbal. İ́n efecto, la oposición de niveles, al igual que la de modos, dentro de la scrie de clasificadores subjetivos del verbo, permite la comunicación del punto de vista personal. list's, además, en consonancia con el condicionaniento de la energia del español que influye sobre las estructuras sintácticas para reorganizarlas y que aqui subraya la preponderancia categorial de lo personal ${ }^{2}$ : apoya la persona del yohablante, el más marcado de los elementos oposicionales entre las personas de la interlocución, ofreciéndole la posibilidad de expresar su opinión subjetiva, subjuntiva con $-s e \mathrm{u}$ optativa con $-r a$, ante el acontecimiento significado por el lexema verbal.

Consecuencia de esta oposición, cuya marca funcional es la actualidad, donde se oponen:

$$
\text { -se actual }+\quad ! \quad-r a \text { inactual - }
$$

es que -se, marcado, implica no ra, mientras que -ra, no marcado, no implica no $-s e^{3}$. Lis lo mismo que decir: todo -se puede quedar sustituido por -ra, pero no al revés 4 .

\section{Conclusiones.}

4.I. Por todo ello, debemos concluir, a nivel de lengua, la posibilidad del triunfo del - $r a$ sobre el -se, muy avanzado en Hispanoamérica, como vimos anteriormente ${ }^{5}$; menos desarrollado, pero en progresión, en la Península, como hemos deducido de nuestra encuesta ${ }^{6}$.

1 Reni, Academira Essiñ̃ot,a: Gramática..., op. cil., § 300.

2 Cir. R. I.APISA: Evolución sintdctica y forma lingüislica inlerior en español. Madrid, CSIC., 1968. Actas del XI Congreso Internacional de Lingüistica y Filologia Románica, v. I, p. I 45 .

3 La oposición binaria opone, ell cronología lógica, un primer elemento marcado por la presencia del rasgo pertincnte, a un segundo elemento que se caracteriza por la ausencia de ese rasgo o marca: $+\underset{7}{\succ}$ La oposición es lógicamente asimétrica e irreversible.

- Lis el fundamento teórico de la exacta afirmación de Cı. I: Kany: Sintaxis..., op. cit., p. 223: "Con el tiempo, es posible que la forma en -se desaparezca completamente». La inversa es lingüisticamente imposible.

- Cfr. §2.2.

- Ividem. 
También a nivel de lengua, la equivalencia de -ra y -se, como afirman muchas de nuestras gramáticas ${ }^{1}$. Pero, en dirección única ${ }^{2}$; 110 puede decirse que las dos formas sean untercambiables»: únicamente -se puede suprimirse en favor de -ra.

4.2. A nivel de discurso, actualización en habla del funcionamiento en lengua, podemos observar la costumbre habitual de los hablantes en este emplco. Así:

r. Los hablantes que usan normalmente la oposición -se/-ra, diferenciando formalmente su respectiva función significativa, el enfoque subjetivo subjuntivo con -se o el enfoque subjetivo optativo con -ra, según la estructura sistematizada que ofrece la lengua ${ }^{3}$. En España todavía se pueden encontrar bastantes 4

2. I,os hablantes que neutralizan la oposición, haciéndose preponderantemente "raistas" o "scistas", según su preferencia por una de las dos formas, aunque no con exclusividad de una sola de cllas. Y teniendo cn cuenta, por un lado, que no podrá aparecer únicamente -se; y, por otra parte, la tendencia a cmplear solamente -ra, muy avanzada cn Iispanoancírica, menos en la Península ${ }^{5}$, posible lingüísticamente por dos razones: por ser $-r a$ el término no marcado de la oposición ${ }^{6}$ y por cl uso de $-r a$ sin alternancia con $-s e$ ?

$3^{\circ}$ Casos de "seísmo" asistemáticos, excepcionales pero perfectamente explicables lingüísticamente por sencilla ultracorrección, especialmente en lablantes que neutralizan la oposición ${ }^{8}$.

4.3. Añadamos, para terminar, que el "raísmo» y el "seísmo" no pueden clazificarse sociolingüísticamente en España. Lo deducimos de unas prospecciones realizadas a distintos niveles culturales: personas

1 Cfr. $\$ 1.3$.

2 Cfr. la anterior nota 3 de la p. 9.

- Cfr. \$3.4.

- Solsre todo en Castilla, muchísimos menos en Andalucia. Is un fenómeno morfosintáctico que en los estudios de geografia lingüistica deberia documentarse al igual que el loísmo, el lésmo y el laismo que, además, a muestro parecer, van muy simultineos.

- Cfr. \$2.2.

- Cir. \$3.4.

- Cir. $\$ 2.4 . y \$ 3.3$.

- Cfr. $\$ 2.3 . y \$ 3.4$. 
cultas con estudios universitarios, administrativos y oficinistas, estudiantes jóvenes, personas menos cultas. En todos y cada uno de estos niveles hemos encontrado hablantes habitualmente diferenciadores de la oposición -se / ra, hiablantes habitualmente "raístas" o "scístis" y hasta algún caso de "sé́smo" asistemático.

Vidal, I, aniquil

Universidad de Sevilla. 